\title{
Predicting episodic memory performance using different biomarkers: results from Argentina- Alzheimer's Disease Neuroimaging Initiative
}

This article was published in the following Dove Press journal:

Neuropsychiatric Disease and Treatment

I3 September 2016

Number of times this article has been viewed

\author{
María Julieta Russo' \\ Gabriela Cohen' \\ Patricio Chrem Mendez' \\ Jorge Campos' \\ Federico E Nahas' \\ Ezequiel I Surace' \\ Silvia Vazquez \\ Deborah Gustafson ${ }^{2,3}$ \\ Salvador Guinjoan' \\ Ricardo F Allegri' \\ Gustavo Sevlever \\ On behalf of the Argentina- \\ Alzheimer's Disease \\ Neuroimaging Initiative \\ group \\ 'Center of Aging and Memory of \\ Neurological Research Institute \\ (FLENI), Buenos Aires, Argentina; \\ ${ }^{2}$ Department of Neurology, State \\ University of New York-Downstate \\ Medical Center, Brooklyn, NY, USA; \\ ${ }^{3}$ Neuropsychiatric Epidemiology \\ Unit, Institute of Neuroscience \\ and Physiology, Sahlgrenska \\ Academy, University of Gothenburg, \\ Gothenburg, Sweden
}

Purpose: Argentina-Alzheimer's Disease Neuroimaging Initiative (Arg-ADNI) is the first ADNI study to be performed in Latin America at a medical center with the appropriate infrastructure. Our objective was to describe baseline characteristics and to examine whether biomarkers related to Alzheimer's disease (AD) physiopathology were associated with worse memory performance.

Patients and methods: Fifteen controls and 28 mild cognitive impairment and 13 AD dementia subjects were included. For Arg-ADNI, all biomarker parameters and neuropsychological tests of ADNI-II were adopted. Results of positron emission tomography (PET) with fluorodeoxyglucose and ${ }^{11} \mathrm{C}$-Pittsburgh compound-B (PIB-PET) were available from all participants. Cerebrospinal fluid biomarker results were available from 39 subjects.

Results: A total of 56 participants were included and underwent baseline evaluation. The three groups were similar with respect to years of education and sex, and they differed in age ( $F=5.10$, $P=0.01)$. Mean scores for the baseline measurements of the neuropsychological evaluation differed significantly among the three groups at $P<0.001$, showing a continuum in their neuropsychological performance. No significant correlations were found between the principal measures (long-delay recall, C-Pittsburgh compound-B scan, left hippocampal volume, and APOE\&4) and either age, sex, or education $(P>0.1)$. Baseline amyloid deposition and left hippocampal volume separated the three diagnostic groups and correlated with the memory performance $(P<0.001)$.

Conclusion: Cross-sectional analysis of baseline data revealed links between cognition, structural changes, and biomarkers. Follow-up of a larger and more representative cohort, particularly analyzing cerebrospinal fluid and brain biomarkers, will allow better characterization of $\mathrm{AD}$ in our country.

Keywords: aging, Alzheimer's disease, amyloid imaging, biomarkers, mild cognitive impairment

\section{Introduction}

Rates of dementia are growing at an alarming rate globally as the world population ages. ${ }^{1}$ Several studies have shown that the overall prevalence of dementia varies widely among countries, mainly related to the influence of cultural and socioeconomic factors. ${ }^{2}$ According to the World Health Organization, in the near future, aging of the population will be more pronounced in underdeveloped countries, while the People's Republic of China, India and Latin America will be the areas more affected by this aging process. Diseases related to the aging process, such as dementia, will be more prevalent in these regions ${ }^{3}$ and it is anticipated that the prevalence and incidence of Alzheimer's disease (AD) will dramatically increase, especially among the group of "oldest old".
Correspondence: María Julieta Russo Center of Aging and Memory of Neurological Research Institute (FLENI), Montañeses 2325, Buenos Aires CI428AQK, Argentina Tel +54 9 I I 57773200 Fax +549 II 57773209 Email jrusso@fleni.org.ar 
There is a continuum of progressive deterioration of cognitive abilities from normal cognition to minimal cognitive impairment (MCI) to dementia syndrome. Early diagnosis of cognitive impairment is highly recommended to help patients and families cope with the disease despite the current lack of an effective curative treatment. Worldwide, numerous new medications are being developed and tried in clinical trials.

Neuroimaging and chemical biomarkers performed at the MCI stage may allow detection of the neurodegenerative process of $\mathrm{AD}$ at an earlier point in the spectrum. ${ }^{4} \mathrm{To}$ achieve this goal, worldwide Alzheimer's Disease Neuroimaging Initiative (ADNI) researchers have established a longitudinal multicenter study designed to develop clinical, imaging, genetic, and biochemical biomarkers for the early detection and tracking of $\mathrm{AD}$. The methodological organization of the Argentina-ADNI is comparable with other worldwide ADNI programs. Since its creation, the Arg-ADNI ${ }^{5}$ has developed a clinical and neuropsychological test battery and has standardized imaging techniques and biomarker procedures in normal subjects, subjects with MCI, and subjects with mild AD. The Arg-ADNI was designed to establish the first worldwide ADNI center in Latin America and to examine: 1) clinical, neuropsychological, and behavioral characteristics; 2) role of risk and protective factors; 3 ) conversion rates from MCI to dementia or normal cognition; and 4) biomarker data on patients with $\mathrm{AD}, \mathrm{MCI}$, and elderly controls. Our objective was to describe the baseline characteristics and to examine whether biomarkers related to $\mathrm{AD}$ physiopathology were associated with worse memory performance.

\section{Patients and methods}

\section{Structure and design of the study}

The methodological organization of the Arg-ADNI is similar to other worldwide ADNI programs. The methods of ArgADNI data collection and study design have been described in detail elsewhere. ${ }^{5}$ The Arg-ADNI team has been enrolling subjects for this study since January 2012. This study was approved by the Medical Ethics Committee of FLENI, Buenos Aires, Argentina. Written informed consent was obtained from all participants and/or their legally acceptable representative.

\section{Subject recruitment and selection of patients}

Enrolled subjects were between 55 and 90 years of age (inclusive), and we admitted subjects with normal cognition (controls), MCI, and $\mathrm{AD}$. We required patients to have a study partner (such as a family member or close friend) to provide an independent evaluation of daily functioning.

Participants underwent a "screening" visit, during which they completed the Mini-Mental State Examination (MMSE), Wechsler's Logical Memory Scale - Revised (LM; Immediate and Delayed Recall), Clinical Dementia Rating (CDR; Global and Sum of Boxes), and Geriatric Depression Scale (GDS). They then underwent a "baseline" visit, at which point they completed the neuropsychological battery, and underwent all test procedures, including neuroimaging and APOE genotyping. According to the ADNI-2 procedure manual, the window from "screening" to "baseline" was 28 days. Participants were then followed longitudinally (6-12 month follow-up visits).

\section{Neuropsychological battery}

At baseline and 1-year follow-up, subjects were evaluated using the Alzheimer's Disease Assessment Scale, Montreal Cognitive Assessment, Clock Drawing Test, Auditory Verbal Learning Test (RAVLT; Total, delayed recall, recognition score), Boston Naming Test, Animal Fluency, Letter Fluency, Trail Making Tests A and B, Neuropsychiatric Inventory Q, GDS, and Functional Assessment Questionnaire (FAQ).

\section{Structural magnetic resonance imaging scans}

All subjects underwent a 3-Tesla magnetic resonance imaging (MRI) scan of the brain including T1, T2, fluidattenuated inversion recovery, and gradient-recalled echo, and diffusion-weighted imaging sequences, and images were then saved in Kodak Carestream System from FLENI for clinical reviewing.

Cortical reconstruction and volumetric segmentation were performed with the automated volumetric output of the FreeSurfer image analysis suite, which is documented and freely available for download online (http://surfer.nmr. mgh.harvard.edu/). FreeSurfer analysis was completed using Version 4.3. The volumetric and cortical thickness measures were corrected for the total intracranial volume. The technical details of these procedures have been described in prior publications. ${ }^{6,7}$

We determined three regions of interest to be measured: the amygdala, hippocampus, and parahippocampal gyrus. The rationale for regions of interest selection was based on which regions have been previously reported as selectively affected by AD in the most of published studies. 


\section{Amyloid positron emission tomography scans (" C-Pittsburgh compound B)}

Positron emission tomography (PET) with fluorodeoxyglucose (FDG) and ${ }^{11} \mathrm{C}$-Pittsburgh compound-B (PIB-PET) was performed in every subject. The synthesis of ${ }^{11} \mathrm{C}$-PIB was performed in the Tracer LAB FXCPRO (General Electric RIS [Radiology Information System] Carestream). After 50 minutes of the endovenous infusion of $10 \mathrm{mCi}$ of ${ }^{11} \mathrm{C}$-PIB, volumetric multislice computerized tomography $(\mathrm{CT})$ brain images were obtained using PET/CT Discovery 690 GE equipment (General Electric RIS - Radiology Information System - Carestream). Images were obtained using a nonuniform attenuation correction with CT. Images were viewed and analyzed by a nuclear medicine physician blinded to the clinical diagnosis. Based on the concentration of activity of ${ }^{11} \mathrm{C}-\mathrm{PIB}$, the degree of cortical retention was classified as positive $(\beta+)$ or negative $(\beta-)$ using visual color linear scales. No other semiquantitative or quantitative assay was performed from the PET images. We also recorded cerebral metabolism using FDG-PET scans. However, results were not considered in this analysis.

\section{APOE genotyping}

In line with other ADNI studies, APOE genotyping for all participants was performed as previously described. ${ }^{5}$ The presence of at least one $\varepsilon 4$ allele was considered as being APOE- $\varepsilon 4$ positive $(\varepsilon 4+)$.

\section{Cerebrospinal fluid biomarkers}

At least $80 \%$ of the participants donated a sample of cerebrospinal fluid (CSF) via a lumbar puncture. FLENI scientists then run CSF biomarkers using enzyme-linked immunosorbent assay kits (Innogenetics; Ghent, Belgium) and our laboratory participates in the ADNI Quality Control Program. CSF markers included the determination of amyloid $\beta 1-42$, total tau protein (t-tau), and tau phosphorylated at position threonine 181 (p-tau181).

\section{Consensus diagnoses}

After each clinical assessment, study neurologists (RFA, MJR, and PC) and neuropsychologists (MEM, MFC, PH, and FT) reviewed the functional, neurologic, and neuropsychological data and reached a consensus regarding the presence or absence of dementia based on the National Institute of Neurological and Communicative Disorders and StrokeAlzheimer's Disease and Related Disorders Association criteria for probable $\mathrm{AD} .{ }^{8}$ Only those who were not diagnosed with dementia were considered for a diagnosis of MCI, which was defined according to Petersen's criteria. ${ }^{9}$

\section{Data analysis}

Statistical analyses were performed with the Statistical Package for the Social Sciences, v19.0 (SPSS Inc., Chicago, IL, USA). Continuous variables were expressed as mean (standard deviation $[\mathrm{SD}]$ ), and categorical variables were expressed as frequencies (\%). The $\chi^{2}$ test was used for comparison of categorical variables. The proportion of subjects "who converted" to dementia was determined (conversion rate $\%=$ total conversions/total MCI subjects $\times 100$ ). Normality was tested using the Shapiro-Wilk test. Assumption of variance homogeneity was assessed using Levene's test. Analysis of variance (ANOVA) or one-way ANOVA was used for comparisons of continuous variables if the samples were distributed normally. If samples were not distributed normally, Kruskal-Wallis one-way ANOVA was used. For multiple comparisons, Bonferroni's post hoc tests were applied. Conversion proportion was estimated. Differences between the mean values were considered statistically significant at $P<0.05$.

The relationship between memory performance measured by long-delay recall on RAVLT and several factors related to AD physiopathology was examined by logistic regression analyses. The full data set was split into two groups to determine which variables were consistent predictors of poor memory performance. Due to skewed distributions of the long-delay recall on the RAVLT, we split the variable at its median to create a dichotomous variable ( $\leq 3$ poor performance or $>3$ good performance). The potential predictors for memory performance were chosen based on the known pathologies and biomarkers of $\mathrm{AD}$ and constituted the main analysis. The independent variables of interest were the result of PIB-PET scan, APOE\&4 carrier, and left hippocampal volume. The primary dependent variable was long-delay free recall on the RAVLT. PIB-PET scan and APOE 4 status were entered into the model as dichotomous variables (ie, positive or negative PIB-PET and presence or absence of at least one $\varepsilon 4$ allele). The models were estimated using the stepwise forward likelihood ratio method.

\section{Results}

\section{Baseline characteristics of study subjects}

Information related to the demographic characteristics of the participant groups are summarized in Table 1. A total of 56 participants were included and underwent baseline evaluation. There were 15 normal controls, 28 subjects with MCI, and 13 with mild AD dementia enrolled in this study. The three groups were similar with respect to years of education and sex, and they differed in age (AD patients 
Table I Demographic characteristics of the participant groups

\begin{tabular}{|c|c|c|c|c|c|c|c|c|c|}
\hline \multirow{2}{*}{$\begin{array}{l}\text { Characteristic } \\
\text { Age, mean } \pm S D \text {, years }\end{array}$} & \multicolumn{2}{|c|}{$\begin{array}{l}\text { Controls } \\
(n=15)\end{array}$} & \multicolumn{2}{|c|}{$\begin{array}{l}\mathrm{MCl} \\
(n=28)\end{array}$} & \multicolumn{2}{|c|}{$\begin{array}{l}A D \\
(n=13)\end{array}$} & \multirow{2}{*}{$\begin{array}{l}F / \chi^{2} \\
5.10\end{array}$} & \multirow{2}{*}{$\begin{array}{l}\text { P-value } \\
0.01\end{array}$} & \multirow{2}{*}{$\begin{array}{l}\mathbf{P}<0.05^{*} \\
\mathrm{a}\end{array}$} \\
\hline & 64.9 & 8.1 & 70.1 & 6.8 & 73.1 & 5.5 & & & \\
\hline Sex (male/female) & $5 / 10$ & & $13 / 15$ & & $6 / 7$ & & 0.76 & 0.68 & \\
\hline Education, mean $\pm S D$, years & 14.5 & 3.4 & 13.1 & 4.4 & 12.2 & 3.9 & 1.26 & 0.29 & \\
\hline Symptom onset, mean \pm SD, years & NA & & 2.9 & 2.7 & 3.2 & 3.7 & 0.08 & 0.78 & \\
\hline \multicolumn{10}{|l|}{ Baseline medication } \\
\hline Total number of medications, mean \pm SD & 2.3 & 2.0 & 4.0 & 1.6 & 4.1 & 1.6 & 5.85 & 0.01 & $\mathrm{a}, \mathrm{c}$ \\
\hline Cholinesterase inhibitors, $\mathrm{n}$ & 0 & & 13 & & 10 & & 17.69 & $<0.001$ & $\mathrm{a}, \mathrm{c}$ \\
\hline Memantine, $\mathrm{n}$ & 0 & & I & & 2 & & 3.60 & 0.17 & \\
\hline Antidepressants, $n$ & I & & 10 & & 4 & & 3.60 & 0.17 & \\
\hline Antipsychotics, n & 0 & & 2 & & 5 & & 2.98 & 0.23 & \\
\hline Carriers APOE\&4, \% & 38.5 & & 36.8 & & 44.4 & & 0.15 & 0.93 & \\
\hline
\end{tabular}

Notes: *Multiple comparisons abbreviated as a: controls differ from subjects with AD, b: subjects with MCl differ from subjects with AD, c: controls differ from subjects with $\mathrm{MCl}$. The $\chi^{2}$ test was used for comparison of categorical variables. One-way ANOVA was used for comparisons of continuous variables.

Abbreviations: $A D$, Alzheimer's disease; $\mathrm{MCl}$, mild cognitive impairment; SD, standard deviation.

were older than the rest). All subjects were white and Spanish speaking. For the $\mathrm{AD}$ and $\mathrm{MCI}$ groups, the mean time from symptom onset to enrollment in Arg-ADNI was $\sim 3$ years. The mean number of total medications per participant in the Arg-ADNI cohort was 3.5 (SD: 1.85). At baseline, 46\% $(\mathrm{n}=12)$ of MCI participants and $80 \%(\mathrm{n}=10)$ of AD subjects received treatment with a cholinesterase inhibitor. One patient in the MCI group and two AD patients were treated also with memantine.

Table 2 shows the mean scores for the screening measures (MMSE, Paragraph Recall, CDR, GDS, and Modified Hachinski Scale). As expected, there was a statistically significant difference in the baseline screening test results in the three groups $(P<0.001)$ for the MMSE, Paragraph Recall, and CDR, with the exception of the GDS and Modified Hachinski Scale scores, in which the difference in all three groups did not reach significance $(P=0.27$ and 0.69$)$.

As shown in Table 3, the mean scores for the baseline measurements of the neuropsychological evaluation differed significantly among the three groups at $P<0.001$, showing a continuum in their neuropsychological performance. Overall, subjects with mild AD had a mean total Montreal Cognitive Assessment score of 16.5, whereas the MCI subjects had a score of 22.9 and the control subjects had a score of 26.6. The subjects with AD performed worse than control and MCI subjects on Clock Drawing Test $(P<0.001)$ and Clock Copying Test $(P=0.01)$, although no differences between the MCI and control groups were observed. The three groups differed significantly in the total number of recalled words, as well as in short-delay (Trial 6) and long-delay (Trial 7) recall from RAVLT, and there was no statistical significance in the number of intrusions in the delayed recall, recognition score, and the number of false alarms. The AD subjects were also impaired in all nonmemory areas tested, including language and executive functions. MCI subjects performed worst compared to control in executive functions domain (Letter Fluency and Trail Making Test). There was no statistical significant difference in behavioral symptoms using the NPI

Table 2 Screening assessments

\begin{tabular}{|c|c|c|c|c|c|c|c|c|c|}
\hline \multirow[t]{2}{*}{ Variables } & \multicolumn{2}{|c|}{ Controls $(n=15)$} & \multicolumn{2}{|c|}{$\operatorname{MCl}(n=28)$} & \multicolumn{2}{|c|}{$\operatorname{AD}(n=13)$} & \multicolumn{3}{|c|}{ ANOVA } \\
\hline & $M$ & SD & $M$ & SD & $M$ & SD & $\boldsymbol{F}$ & $P$-value & $\mathbf{P}<0.05^{*}$ \\
\hline $\mathrm{MMSE}^{13}$ & 29.2 & 1.5 & 28.6 & 1.3 & 22.0 & 3.2 & 60.16 & $<0.001$ & $\mathrm{a}, \mathrm{b}$ \\
\hline LM immediate recall' & 10.2 & 2.6 & 6.3 & 3.3 & 2.3 & 1.8 & 27.42 & $<0.001$ & $a, b, c$ \\
\hline LM delayed recall $^{14}$ & 7.9 & 2.2 & 3.8 & 3.5 & 0.5 & I.I & 25.68 & $<0.001$ & $a, b, c$ \\
\hline GDS- $15^{15}$ & 1.7 & 1.5 & 2.4 & 2.3 & 1.5 & I.I & 1.35 & 0.27 & \\
\hline CDR - screening ${ }^{16}$ & 0.0 & 0.1 & 0.5 & 0.0 & 0.7 & 0.3 & 89.72 & $<0.001$ & $a, b, c$ \\
\hline CDR-SOB - screening ${ }^{16}$ & 0.0 & 0.1 & 1.0 & 0.8 & 3.7 & 1.6 & 55.62 & $<0.001$ & $a, b, c$ \\
\hline Modified Hachinski ${ }^{17}$ & 0.3 & 0.8 & 0.2 & 0.5 & 0.3 & 0.5 & 0.37 & 0.69 & \\
\hline
\end{tabular}

Notes: *Multiple comparisons abbreviated as a: controls differ from subjects with AD, b: subjects with $\mathrm{MCl}$ differ from subjects with AD, c: controls differ from subjects with $\mathrm{MCl}$. ( 2012 Used with the permission of the Alzheimer's Disease Neuroimaging Initiative. ${ }^{13-17}$

Abbreviations: AD, Alzheimer's disease; MCl, mild cognitive impairment; MMSE, Mini-Mental State Examination; LM, logical memory; GDS, Geriatric Depression Scale; CDR, Clinical Dementia Rating; CDR-SOB, Clinical Dementia Rating-Sum of Boxes; ANOVA, analysis of variance; SD, standard deviation. 
Table 3 Baseline assessments

\begin{tabular}{|c|c|c|c|c|c|c|c|c|c|}
\hline \multirow[t]{2}{*}{ Variables } & \multicolumn{2}{|c|}{ Controls $(n=15)$} & \multicolumn{2}{|c|}{$\mathrm{MCl}(\mathrm{n}=28)$} & \multicolumn{2}{|c|}{$\operatorname{AD}(n=13)$} & \multicolumn{3}{|c|}{ ANOVA } \\
\hline & $M$ & SD & $M$ & SD & $M$ & SD & $\boldsymbol{F}$ & $P$-value & $P<0.05 *$ \\
\hline $\mathrm{MoCA}^{18}$ & 26.6 & 4.0 & 22.9 & 3.5 & 16.5 & 3.9 & 24.22 & $<0.001$ & $a, b, c$ \\
\hline Clock Drawing ${ }^{19}$ & 5.0 & 0.0 & 4.4 & 1.0 & 2.77 & 1.6 & 16.31 & $<0.001$ & $a, b$ \\
\hline Clock Copying ${ }^{19}$ & 5.0 & 0.0 & 5.0 & 0.2 & 4.4 & 1.2 & 4.76 & 0.01 & $a, b$ \\
\hline RAVLT Total ${ }^{20}$ & 42.9 & 8.3 & 30.4 & 8.6 & 20.2 & 6.8 & 26.20 & $<0.001$ & $a, b, c$ \\
\hline RAVLT immediate recall ${ }^{20}$ & 9.3 & 3.7 & 4.40 & 2.3 & 2.0 & 1.8 & 25.14 & $<0.001$ & $a, b, c$ \\
\hline RAVLT delayed recall ${ }^{20}$ & 8.2 & 2.4 & 3.3 & 2.5 & 0.4 & 0.8 & 47.51 & $<0.001$ & $a, b, c$ \\
\hline Intrusions Delayed ${ }^{20}$ & 0.5 & 0.6 & 0.9 & 1.6 & 0.1 & 0.3 & 2.05 & 0.14 & \\
\hline RAVLT Recognition Score ${ }^{20}$ & 8.5 & 6.3 & 5.4 & 5.3 & 4.7 & 4.2 & 2.11 & 0.13 & \\
\hline False Alarms ${ }^{20}$ & 0.1 & 0.3 & 1.1 & 2.1 & 1.5 & 1.9 & 2.75 & 0.07 & \\
\hline Boston Naming Test ${ }^{21}$ & 26.0 & 7.6 & 25.5 & 5.8 & 20.0 & 5.5 & 4.12 & 0.02 & $a, b$ \\
\hline Category Fluency (animals) & 21.5 & 3.4 & 17.6 & 3.2 & 12.1 & 4.0 & 26.21 & $<0.001$ & $a, b, c$ \\
\hline Letter Fluency (“p”) $)^{22}$ & 19.5 & 4.3 & 15.6 & 5.3 & 13.5 & 5.0 & 5.29 & 0.01 & a \\
\hline Trail Making A (seconds) ${ }^{23}$ & 33.8 & 8.7 & 51.1 & 21.4 & 125.5 & 121.4 & 9.22 & $<0.001$ & $\mathrm{a}, \mathrm{b}$ \\
\hline Trail Making B (seconds) ${ }^{23}$ & 75.8 & 29.1 & 143.4 & 86.7 & 288.9 & 97.0 & 26.77 & $<0.001$ & $a, b, c$ \\
\hline $\mathrm{NP}^{24}$ & 0.4 & 0.7 & 4.1 & 5.5 & 9.5 & 15.6 & 2.77 & 0.08 & \\
\hline $\mathrm{FAQ}^{25}$ & 0.0 & 0.0 & 3.2 & 0.4 & 12.8 & 9.8 & 15.05 & $<0.001$ & $a, b$ \\
\hline
\end{tabular}

Notes: *Multiple comparisons abbreviated as a: controls differ from subjects with AD, b: subjects with MCI differ from subjects with AD, c: controls differ from subjects with $\mathrm{MCl}$. (C) 2012 Used with the permission of the Alzheimer's Disease Neuroimaging Initiative. ${ }^{18-25}$

Abbreviations: AD, Alzheimer's disease; MCl, mild cognitive impairment; MoCA, Montreal Cognitive Assessment; RAVLT, Auditory Verbal Learning Test (Total, delayed recall, recognition score); NPI, Neuropsychiatric Inventory; FAQ, Functional Assessment Questionnaire; ANOVA, analysis of variance; SD, standard deviation.

reported by family members in the three groups, although we saw a trend toward more behavioral symptoms in the $\mathrm{AD}$ group compared to the rest. As expected, we observed a significant difference in the daily functions using FAQ with more impairment in the $\mathrm{AD}$ group compared to the other groups and no significant difference in performance of activities between the control and MCI patients. Interestingly, for the MCI group, we found mild compromise in activities of daily living, mainly in sophisticated instrumental activities using the FAQ (writing checks, paying bills, keeping financial records; assembling tax or business records; and remembering appointments, family occasions, and medications). These data are not shown.

\section{Biomarkers results of study subjects}

Table 4 shows the results of MRI and CSF biomarkers. Regarding volumetric MRI measurements, all regions of interest measured (amygdala, hippocampus, and parahippocampal gyrus) were significantly different $(P<0.05)$ in control subjects compared to MCI and AD subjects, but interestingly, there were no differences between the MCI and AD groups. As outlined in the ADNI protocol, we then

Table 4 Biomarkers results

\begin{tabular}{|c|c|c|c|c|c|c|c|c|c|}
\hline Variables & Control & & $\mathrm{MCl}$ & & AD & & $\chi^{2}$ & $P$-value & $\mathbf{P}<0.05^{*}$ \\
\hline Volumetric MRI measurements $\left(\mathrm{mm}^{3}\right)$, mean $\pm \mathrm{SD}$ & $(n=15)$ & & $(n=28)$ & & $(n=13)$ & & & & \\
\hline Left hippocampus & $4,127.7$ & 727.2 & $3,300.6$ & 730.6 & $2,867.5$ & 696.6 & 16.12 & $<0.001$ & a, c \\
\hline Left amygdala & $\mathrm{I}, 440.4$ & 324.2 & I, 176.5 & 297.0 & 989.8 & 251.9 & 12.78 & $<0.001$ & a, c \\
\hline Left parahippocampal gyrus & $3,806.6$ & 639.1 & $3,290.9$ & 842.0 & $2,7 \mid 4.0$ & 645.8 & 11.45 & $<0.001$ & $\mathrm{~b}$ \\
\hline Right hippocampus & $4,161.0$ & 743.4 & $3,235.7$ & 627.0 & $2,782.0$ & 618.3 & $|8.6|$ & $<0.001$ & $\mathrm{a}, \mathrm{c}$ \\
\hline Right amygdala & $\mathrm{I}, 605.6$ & 339.8 & $\mathrm{I}, 243.5$ & 259.7 & I, 176.9 & 252.4 & 12.94 & $<0.001$ & $\mathrm{a}, \mathrm{c}$ \\
\hline Right parahippocampal gyrus & $4,161.8$ & 813.7 & $3,498.6$ & 676.3 & $2,945.6$ & 774.5 & 15.02 & $<0.001$ & a, c \\
\hline CSF biomarkers $(\mathrm{pg} / \mathrm{mL})$, mean $\pm \mathrm{SD}$ & $(n=10)$ & & $(n=21)$ & & $(n=8)$ & & & & \\
\hline Abeta & 824.2 & 256.5 & 678.8 & 353.3 & 423.8 & 269.3 & 8.24 & 0.02 & $a, b$ \\
\hline Tau total & 207.4 & 76.3 & 379.9 & 264.1 & 596.4 & 261.2 & 8.67 & 0.01 & $a, b$ \\
\hline $\mathrm{p}-\mathrm{Tau}$ & 37.5 & 12.5 & 61.9 & 42.7 & 78.5 & 27.1 & 5.79 & 0.06 & \\
\hline Positive amyloid PET, \% & 14.2 & & 50.0 & & 83.3 & & $|2.4|$ & $<0.001$ & $a, b, c$ \\
\hline
\end{tabular}

Notes: *Multiple comparisons abbreviated as a: controls differ from subjects with AD, b: subjects with $M C l$ differ from subjects with AD, c: controls differ from subjects with $\mathrm{MCl}$.

Abbreviations: AD, Alzheimer's disease; $\mathrm{MCl}$, mild cognitive impairment; $\mathrm{MRI}$, magnetic resonance imaging; CSF, cerebral spinal fluid; PET, positron emission tomography. 


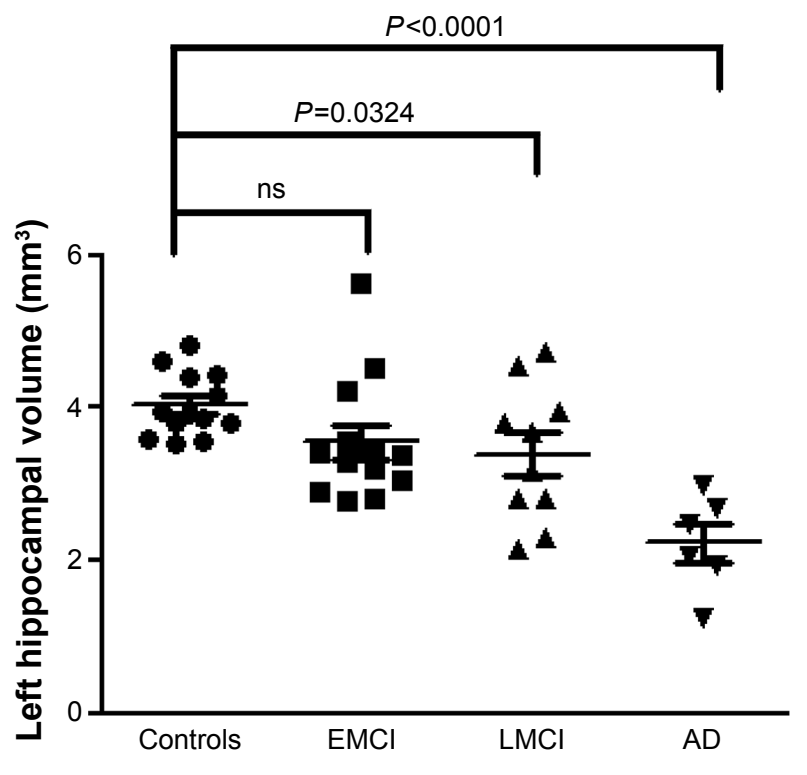

Figure I MRI volumetry of left hippocampus in Argentina-ADNI subjects. Abbreviations: $A D$, Alzheimer's disease; $\mathrm{EMCl}$, early mild cognitive impairment; $\mathrm{LMCl}$, late mild cognitive impairment; ns, nonsignificant; MRI, magnetic resonance imaging; ADNI, Alzheimer's Disease Neuroimaging Initiative.

considered the $\mathrm{MCI}$ group and split it into early versus late MCI, and we found a significantly lower hippocampal volume in subjects with late MCI compared to controls, and no statistical difference between controls and those with early MCI (Figure 1).

CSF results were available from ten control subjects, 21 patients with MCI, and eight patients with mild AD. Baseline CSF A $\beta-42$ levels decreased significantly across the groups $(P<0.05)$ with a slope of values from the control subjects to $\mathrm{MCI}$ and $\mathrm{AD}$ patients. Baseline t-tau levels increased significantly $(P<0.05)$ and $p$-tau levels increased marginally $(P=0.06)$ across the groups. There were no differences between the controls and MCI groups in CSF biomarkers. All subjects underwent an amyloid PET scan. The following percentages of subjects had an amyloid positive $(\mathrm{A} \beta+) \mathrm{PET}$ scans at baseline: normal controls: $14.2 \%$, subjects with MCI: $50 \%$, and subjects with mild AD: $83.3 \%$, with a significant difference in percentage distribution across diagnostic groups ( $P=0.002$; Figure 2$)$.

\section{Prediction of memory performance main analyses}

A logistic regression analysis was conducted for successfully predicting the memory performance of the patient population using PIB-PET scan, left hippocampal volume, and APOE\&4 status as predictors. No significant correlations were found between the principal measures (long-delay recall, PIB-PET scan, left hippocampal volume, and APOE\&4) and either

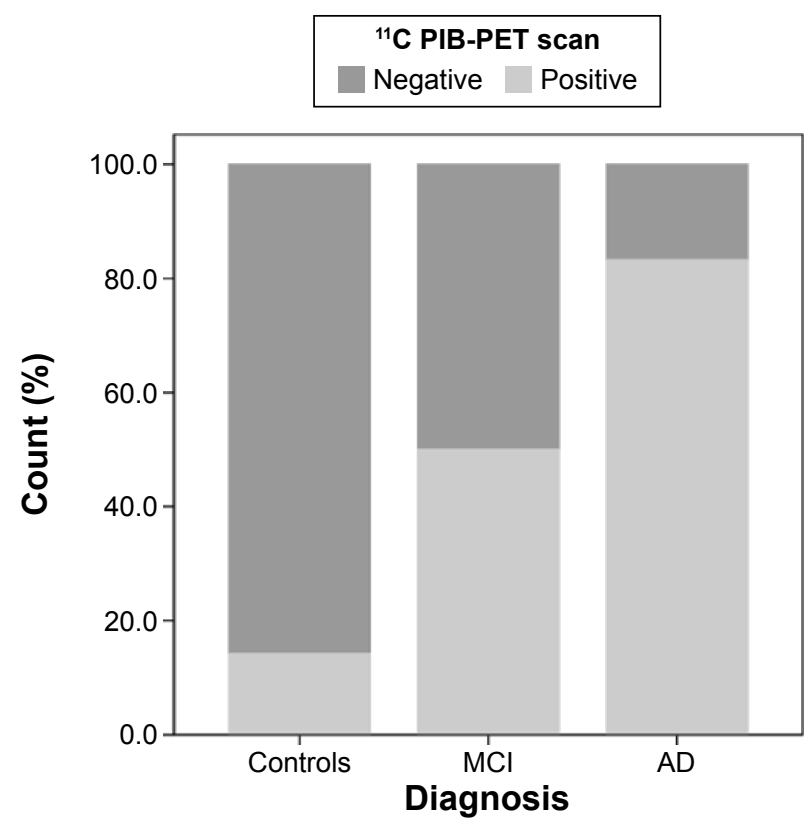

Figure 2 Percentage of amyloid positive $(A \beta+)$ and negative $(A \beta-)$ PET scans at baseline in Arg-ADNI.

Abbreviations: $A D$, Alzheimer's disease; $M C l$, mild cognitive impairment; $P E T$, positron emission tomography; Arg-ADNI, Argentina-Alzheimer's Disease Neuroimaging Initiative; " C PIB-PET, "C-Pittsburgh compound-B.

age, sex, or education $(P>0.1)$. Consequently, these measures were not considered for further analysis. A test of the full model against a constant-only model was statistically significant, indicating that the predictors as a set reliably distinguished between individuals having a long-delay recall on RAVLT of $<3$ with those having a higher score $\left(\chi^{2}=14.802\right.$, $P<0.001$ with $d f=1$ ).

Nagelkerke's $R^{2}$ of 0.711 indicated a moderately strong relationship between prediction and grouping (Table 5). Prediction success overall was $87.5 \%$ (85\% for good performance and $90 \%$ for poor performance). The Wald criterion demonstrated that only PIB-PET scan and left hippocampal volume made a significant contribution to prediction $(P=0.01)$. APOE $\varepsilon 4$ status was not a significant predictor (Figure 3).

\section{Discussion}

This report presents the clinical and biomarkers profile of the baseline evaluation of Arg-ADNI. Our study is a longitudinal prospective cohort study of 56 subjects, aged 55-90 years recruited at a single study site in Argentina. Our institution is the only medical center in the region with the infrastructure to perform the biomarkers and PET study with Pittsburg compound. All the procedures and follow-up were performed at our center with highly standardized protocols and strictly adhered to worldwide study sites. By following the ADNI-II 
Table 5 Summary of logistic regression analysis predicting memory performance

\begin{tabular}{|c|c|c|c|c|c|c|c|}
\hline Model terms & B & SE & Wald & $P$-value & $-2 L L$ & Nagelkerke's $R^{2}$ & $\begin{array}{l}\text { Percentage } \\
\text { correct }\end{array}$ \\
\hline Intercept & 0.0 & 0.3 & 0.00 & 1.00 & 55.452 & & 50.0 \\
\hline Step I: PIB-PET positive & -2.833 & 0.812 & 12.184 & $<0.001$ & 39.802 & 0.432 & 80.0 \\
\hline Step 2: PIB-PET positive & -2.659 & 1.038 & 6.567 & 0.01 & 25.000 & 0.711 & 87.5 \\
\hline Left hippocampal volume & -0.003 & 0.001 & 6.747 & 0.01 & & & \\
\hline
\end{tabular}

Notes: The dependent variable in this analysis was memory performance measured by long-delay recall on RAVLT coded so that $0=>3$ (good performance) and $\mathrm{I}=\leq 3$ (poor performance). The independent variables of interest were the result of PIB-PET scan, APOE $\varepsilon 4$ carrier, and left hippocampal volume. The models were estimated using the stepwise forward LR method.

Abbreviations: PIB-PET, "C-Pittsburgh compound-B; RAVLT, Auditory Verbal Learning Test (Total, delayed recall, recognition score); LR, likelihood ratio.

recruitment protocol, Arg-ADNI represents a similar cohort to other clinical studies. ${ }^{4}$

The summary of the baseline characteristics of the Arg-ADNI subjects showed that the three groups were comparable to subjects recruited at other worldwide ADNI sites. ${ }^{4}$ Neuropsychology, structural, and molecular measures showed a continuum between the three groups, with $\mathrm{AD}$ being the worst group, controls better, and MCI subjects showing an intermediate status (especially, long-delay recall, hippocampal volume, amyloid deposition measured by PIBPET and CSF). Furthermore, the amyloid deposition and hippocampal atrophy data highly correlated with the memory performance. Similar findings have arisen in related studies supporting the use of hippocampal volume and amyloid deposition as reliable and valid surrogate measures in $\mathrm{AD}$ clinical drug trials. ${ }^{10,11}$

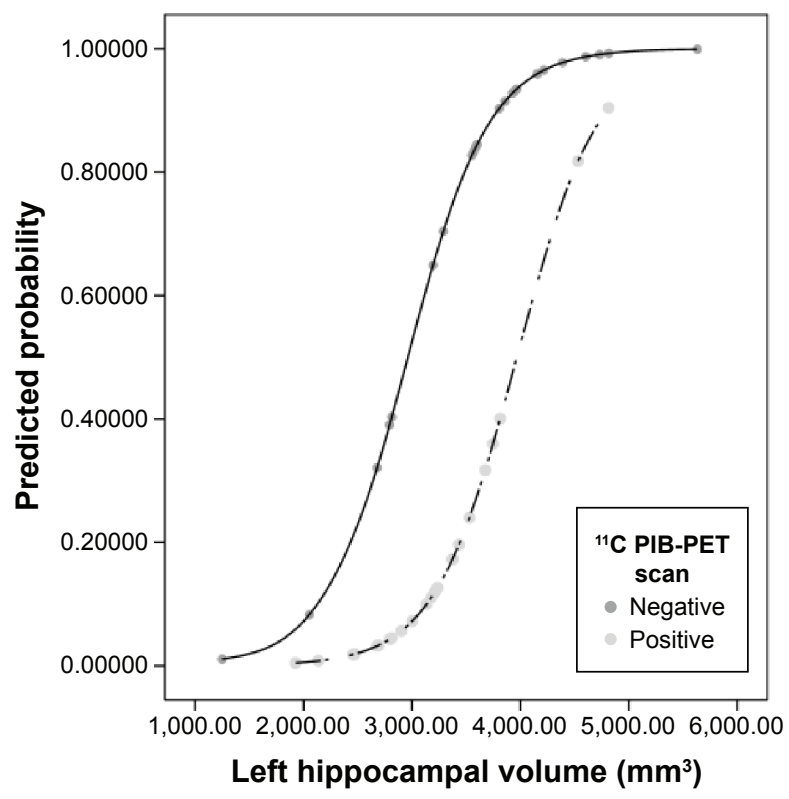

Figure 3 Logistic regression results.

Notes: The plot of Logistic Regression Model displays the probability of the outcome (memory performance) versus significant predictor variables (PIB-PET scans and left hippocampal volume), with the other variables held constant. Abbreviation: "C PIB-PET, " C-Pittsburgh compound-B.
We observed that almost half the patients in the MCI group received medication with cholinesterase inhibitor. Treatment with this group of medications for MCI is currently out off label for this diagnosis, and it is interesting to observe that the clinical practice of neurologists in our center that did not differ from that reported in other ADNI sites. ${ }^{4,12}$ Very few patients in this group of patients with only mild $\mathrm{AD}$ were treated with memantine, which is consistent with the current indication of this drug restricted to moderate-tosevere AD.

\section{Conclusion}

Limitations of our study are, in the first place, the relatively small cohort of subjects included in this first pilot study. This is in part due to funding constraints for research in developing countries. Another limitation is that our study was performed in a unique, single-specialized medical center, and thus could be less representative of the real population of patients of our region. All investigators from this pilot team hope to develop a new pathway to create a multisite ADNI in Argentina, with the intention of using these preliminary results obtained to seek continued funding from local and international agencies. Follow-up of a larger and more representative cohort, particularly related to CSF and brain biomarkers, will allow for better characterization of AD in our country, and perhaps point to unique environmental factors influencing brain health in South America. ${ }^{5}$

\section{Acknowledgments}

Members of the Argentina-Alzheimer's Disease Neuroimaging Initiative include María Eugenia Martin, Horacio Martinetto, Fernando Ventrice, Alejandra Amengual, María Florencia Clarens, Paula Harris, Fernanda Tapajoz, and Miguel Riudavets.

\section{Disclosure}

The authors report no conflicts of interest in this work. 


\section{References}

1. Kalaria RN, Maestre GE, Arizaga R, et al. Alzheimer's disease and vascular dementia in developing countries: prevalence, management, and risk factors. Lancet Neurol. 2008;7(9):812-826.

2. Suh GH, Shah A. A review of the epidemiological transition in dementia - cross-national comparisons of the indices related to Alzheimer's disease and vascular dementia. Acta Psychiatr Scand. 2001;104(1):4-11.

3. World Health Organization. Dementia: a public health priority. 2012:112. Available from: http://apps.who.int/iris/bitstream/10665/7 5263/1/9789241564458_eng.pdf?ua=1. Accessed May 23, 2016.

4. Petersen RC, Aisen PS, Beckett LA, et al. Alzheimer's Disease Neuroimaging Initiative (ADNI): clinical characterization. Neurology. 2010; 74(3):201-209.

5. Russo MJ, Gustafson D, Vázquez S, et al; Argentina-Alzheimer's Disease Neuroimaging Initiative. Creation of the Argentina-Alzheimer's Disease Neuroimaging Initiative. Alzheimers Dement. 2014;10(1 Suppl): S84-S87.

6. Dale AM, Fischl B, Sereno MI. Cortical surface-based analysis. I. Segmentation and surface reconstruction. Neuroimage. 1999;9(2): 179-194.

7. Fischl B, van der Kouwe A, Destrieux C, et al. Automatically parcellating the human cerebral cortex. Cereb Cortex. 2004;14(1):11-22.

8. McKhann GM, Drachman D, Folstein M, Katzman R, Price D, Stadlan EM. Clinical diagnosis of Alzheimer's disease: report of the NINCDS-ADRDA Work Group under the auspices of Department of Health and Human Services Task Force on Alzheimer's Disease. Neurology. 1984;34:939-944.

9. Petersen RC, Stevens JC, Ganguli M, Tangalos EG, Cummings JL, DeKosky ST. Practice parameter: early detection of dementia: mild cognitive impairment (an evidence-based review). Report of the Quality Standards Subcommittee of the American Academy of Neurology. Neurology. 2001;56:1133-1142.

10. Chételat G, Villemagne VL, Pike KE, et al; Australian Imaging Biomarkers and Lifestyle Study of ageing (AIBL) Research Group. Independent contribution of temporal $\beta$-amyloid deposition to memory decline in the pre-dementia phase of Alzheimer's disease. Brain. 2011; 134(Pt 3):798-807.

11. Pike KE, Ellis KA, Villemagne VL, et al. Cognition and $\beta$-amyloid in preclinical Alzheimer's disease: data from the AIBL study. Neuropsychologia. 2011;49(9):2384-2390.

12. Schneider LS, Insel PS, Weiner MW; Alzheimer's Disease Neuroimaging Initiative. Treatment with cholinesterase inhibitors and memantine of patients in the Alzheimer's Disease Neuroimaging Initiative. Arch Neurol. 2011;68(1):58-66.
13. Folstein MF, Folstein SE, McHugh PR. "Mini mental state". A practical method for grading the cognitive state of patients for the clinician. JPsychiatr Res. 1975;12:189-198. doi:10.1016/0022-3956(75)90026-6

14. Wechsler D. WMS-R Wechsler Memory Scale-Revised Manual. New York; NY: The Psychological Corporation, Harcourt Brace Jovanovich, Inc.; 1987.

15. Yesavage JA, Brink TL, Rose TL, et al. Development and validation of a geriatric depression screening scale: a preliminary report. J Psychiatr Res. 1982;17(1):37-49.

16. Hughes CP, Berg L, Danziger WL, Coben LA, Martin RL. A new clinical scale for the staging of dementia. Br J Psychiatry. 1982;140:566-572. Available at: http://www.ncbi.nlm.nih.gov/pubmed/7104545. Accessed September 30, 2014.

17. Rosen WG, Terry RD, Fuld PA, Katzman R, Peck A. Pathological verification of ischemic score in differentiation of dementias. Ann Neurol. 1980;7:486-488. doi:10.1002/ana.410070516

18. Nasreddine ZS, Phillips NA, Bédirian V, et al. The Montreal Cognitive Assessment, MoCA: a brief screening tool for mild cognitive impairment. J Am Geriatr Soc. 2005;53(4):695-699. doi:10.1111/j.1532-5415. 2005.53221.x

19. Freedman M, Leach L, Kaplan E, Winocur G, Shulman KI, Delis DC. Clock Drawing: A Neuropsychological Analysis. New York: Oxford University Press; 1994.

20. Rey A. L'examen clinique en psychologie. Paris: Presses Universitaires de France; 1964.

21. Kaplan E, Goodglass H, Weintraub S. The Boston Naming Test. Philadelphia: Lea \& Febiger; 1983.

22. Butters N, Granholm E, Salmon DP, Grant I, Wolfe J. Episodic and semantic memory: a comparison of amnesic and demented patients. J Clin Exp Neuropsychol. 1987;9(5):479-497. doi:10.1080/ 01688638708410764

23. Reitan RM. Validity of the Trail Making Test as an indicator of organic brain damage. Percept Mot Skills. 1958;8:271-276. doi:10.2466/ pms.1958.8.3.271

24. Kaufer DI, Cummings JL, Ketchel P, et al. Validation of the NPI-Q, a brief clinical form of the Neuropsychiatric Inventory. JNeuropsychiatry Clin Neurosci. 2000;12(2):233-239. Available at: http://www.ncbi.nlm. nih.gov/pubmed/11001602. Accessed June 3, 2014.

25. Pfeffer RI, Kurosaki TT, Harrah CH, Chance JM, Filos S. Measurement of functional activities in older adults in the community. J Gerontol. 1982; 37:323-329. doi:10.1093/geronj/37.3.323
Neuropsychiatric Disease and Treatment

\section{Publish your work in this journal}

Neuropsychiatric Disease and Treatment is an international, peerreviewed journal of clinical therapeutics and pharmacology focusing on concise rapid reporting of clinical or pre-clinical studies on a range of neuropsychiatric and neurological disorders. This journal is indexed on PubMed Central, the 'PsycINFO' database and CAS,
Dovepress

and is the official journal of The International Neuropsychiatric Association (INA). The manuscript management system is completely online and includes a very quick and fair peer-review system, which is all easy to use. Visit http://www.dovepress.com/testimonials.php to read real quotes from published authors. 